\title{
O desenvolvimento de competências do docente fisioterapeuta de uma instituição pública do estado de Goiás, Brasil e suas abordagens interdisciplinares e atenção à saúde pública
}

The development of competences of the physiotherapy teacher of a public institution in the state of Goiás, Brazil and its interdisciplinary approaches and attention to public health

El desarrollo de las competencias del professor de fisioterapia de uma institución pública del estado de Goiás, Brasil e sus enfoques interdisciplinarios y atención a la salud pública

Recebido: 13/05/2021 | Revisado: 20/05/2021 | Aceito: 25/05/2021 | Publicado: 09/06/2021

Heliny Alves do Santos

ORCID: https://orcid.org/0000-0002-2685-8182 Universidade Estadual de Goiás, Brasil

E-mail: helinyalvesdossantos@gmail.com

Tássio Moreira Peres

ORCID: https://orcid.org/0000-0002-1167-8955 Universidade Estadual de Goiás, Brasil

E-mail: tassioperes@gmail.com

Clarice Fernandes Pimentel

ORCID: https://orcid.org/0000-0002-9089-2166 Universidade Estadual de Goiás, Brasil

E-mail: claricefernandespimentel@gmail.com

Hygor Willian de Oliveira

ORCID: https://orcid.org/0000-0002-0895-2175 Universidade Estadual de Goiás, Brasil E-mail: hygorfgb@gmail.com Jessé Castelo Souza Santana

ORCID: https://orcid.org/0000-0002-1955-1422 Universidade Estadual de Goiás, Brasil

E-mail: jessecasteloss@gmail.com

Luiz Henrique Leite Silva

ORCID: https://orcid.org/0000-0002-1429-1887 Universidade Estadual de Goiás, Brasil E-mail: luislhls@hotmail.com Franassis Barbosa de Oliveira

ORCID: https://orcid.org/0000-0001-5145-1491 Universidade Estadual de Goiás, Brasil

E-mail: franassis_oliveira@yahoo.com.br

\begin{abstract}
Resumo
Objetivo: Analisar o desenvolvimento de competências do docente fisioterapeuta de uma instituição pública do estado de Goiás, Brasil, e se suas estratégias de atuação acadêmica têm abordagens interdisciplinares e voltadas à Saúde Pública. Método: Foi realizado um estudo do tipo transversal, de abordagem quantitativa e qualitativa, por meio de um questionário misto aos professores, com perguntas abertas, fechadas e de múltipla escolha. A amostra foi composta de 24 professores da Universidade Estadual de Goiás, Brasil. Resultados e discussão: A prática docente no ensino superior no Brasil, de maneira geral, tende a iniciar-se sem preparação prévia e de modo intuitivo. Os entrevistados apontam a fisioterapia como uma área em crescimento, contudo há um impasse com relação ao retorno financeiro. Entende-se que a fisioterapia é uma profissão essencial nos três níveis de atenção à saúde, apesar de ainda ser associada somente ao nível terciário. A formação acadêmica interfere de forma incisiva na forma de atuação destes profissionais, no que se refere a saúde pública é fundamental o conhecimento sobre o sistema. A perspectiva é de um atendimento e formação mais atrelados ao modelo biopsicossocial e a tecnologia. Conclusão: A atuação prática da fisioterapia é reflexo direto do aprendizado acadêmico e por isso os docentes da área devem e tentam buscar melhoras no exercício do ensino, uma vez que as práticas e manejos tem repercussão direta na sociedade, principalmente no tópico da Saúde Pública.
\end{abstract}

Palavras-chave: Fisioterapia; Ensino; Saúde Pública; Interdisciplinaridade. 


\begin{abstract}
Objective: To analyze the development of competencies of physical therapist professors in a public institution in the state of Goiás, Brazil, and whether their academic performance strategies have interdisciplinary and public health oriented approaches. Method: A cross-sectional study with a quantitative and qualitative approach was carried out using a mixed questionnaire for professors, with open, closed and multiple choice questions. The sample was composed of 24 professors from the State University of Goiás, Brazil. Results and discussion: Teaching practice in higher education in Brazil, in general, tends to begin without previous preparation and intuitively. The interviewees point to physical therapy as an area of growth, but there is a lack of financial recognition. It is understood that physical therapy is an essential profession in the three levels of health care, although it is still associated only with the tertiary level. The academic training interferes incisively in the way these professionals act, in what refers to public health, the knowledge about the system is fundamental. The perspective is of a care and training more linked to the biopsychosocial model and technology. Conclusion: The practical performance of physical therapy is a direct reflection of academic learning and therefore the teachers of the area must and try to seek improvements in the exercise of teaching, since the practices and management have direct repercussions on society, especially on the topic of public health.
\end{abstract}

Keywords: Physiotherapy; Teaching; Public health; Interdisciplinarity.

\title{
Resumen
}

Objetivo: Analizar el desarrollo de competencias del docente fisioterapeuta de una institución pública en el estado de Goiás, Brasil, y si sus estrategias de desempeño académico tienen enfoques interdisciplinarios y enfocados a la Salud Pública. Método: Se realizó un estudio transversal, con un enfoque cuantitativo y cualitativo, mediante un cuestionario mixto a los docentes, con preguntas abiertas, cerradas y de opción múltiple. La muestra estuvo compuesta por 24 profesores de la Universidad Estatal de Goiás, Brasil. Resultados y discusión: La práctica docente en la educación superior en Brasil, en general, tiende a iniciarse sin preparación previa y de manera intuitiva. Los encuestados señalan a la fisioterapia como un área en crecimiento, sin embargo, existe un callejón sin salida con respecto al rendimiento financiero. Se entiende que la fisioterapia es una profesión imprescindible en los tres niveles asistenciales, aunque todavía está asociada únicamente al nivel terciario. La educación académica tiene una incisiva influencia en la forma en que estos profesionales trabajan, en lo que a salud pública se refiere, el conocimiento del sistema es fundamental. La perspectiva es de servicio y formación más vinculados al modelo y tecnología biopsicosocial. Conclusión: La realización práctica de la fisioterapia es un reflejo directo del aprendizaje académico y por ello los docentes del área deben e intentar buscar mejoras en el ejercicio de la docencia, ya que las prácticas y la gestión tienen un impacto directo en la sociedad, especialmente en el tema de la Salud Pública.

Palabras clave: Fisioterapia; Enseñanza; Salud pública; Interdisciplinariedad.

\section{Introdução}

O termo Saúde Pública reverbera na sociedade muitas vezes de maneira equivocada, uma vez que pode ser entendido como algo de dever unicamente do Estado. Entretendo, a Saúde Pública depende tanto das ações governamentais quanto da ação coletiva da sociedade civil e dessa forma é definida como uma prática social interdisciplinar, ao englobar tanto a prevenção de doença quanto a promoção de saúde (OPAS/OMS, 2000).

Ainda nesse contexto, é relevante também diferenciar o termo Saúde Pública de Saúde Coletiva. Nesse aspecto (Souza, 2014) destaca que a pública toma como objetivo os problemas da saúde, enquanto a coletiva trata das necessidades sociais da saúde e considera a situação como um processo social. Outrossim, em todos os casos é validado a necessidade de um comprometimento conjunto da esfera governamental, social e gestora.

No Brasil, a saúde é um direito resguardado pelo Art. 196 da Constituição Federal enunciando que "a saúde é direito de todos e dever do Estado(...)". Logo essa é a base do Sistema Único de Saúde (SUS), instituído pela Lei 8.080 e tem como gestor, no âmbito da União, o Ministério da Saúde (Souza, 2002). O SUS é pautado nos princípios da universalidade, integridade e equidade, logo engloba toda a população em ações de prevenção e de reabilitação ( Carvalho, 2013).

Ademais, o funcionamento do SUS depende do trabalho de diversos profissionais de distintas áreas. Dentre esses está o fisioterapeuta, profissional que tem condição de atuar nos três níveis de atenção à saúde: o nível primário, que está relacionado com estratégias de prevenção; o secundário, que envolve o diagnóstico e tratamento de doenças e o terciário, fase de atendimento especializado. Portanto, este modelo de atuação profissional vai de encontro com a proposta e os princípios do 
SUS (Bispo Júnior, 2010). Apesar desses fatores a fisioterapia ainda é muito atrelada com a reabilitação, contudo ela tem papel fundamental na prevenção primária.

No trabalho realizado por Carvalho, Tomaz e Tavares em 2018 ( Carvalho et al., 2018) foi investigado a relação da interdisciplinaridade na formação acadêmica, a partir da ideia de que a fragmentação disciplinar corrobora com um modelo de fisioterapia assistencialista e sem grande atenção à prevenção da doença e a promoção da saúde. Em contrapartida, a interdisciplinaridade constrói um novo saber e propaga uma formação acadêmica voltada para todos os níveis de atenção à saúde.

A formação acadêmico-profissional do fisioterapeuta the dá a capacidade de avaliar, prevenir e promover saúde. Dessa forma, lhe é garantida a competência de operar em programas de atenção básica (Maia et al., 2015). O crescente número de fisioterapeutas atuando na atenção básica mostra-se relacionado com a vinculação da formação e atuação voltadas para às políticas nacionais de saúde (Tavares et al., 2018). Diante do exposto, este trabalho tem o objetivo de analisar o desenvolvimento de competências do docente fisioterapeuta de uma instituição pública do estado de Goiás, Brasil, e se suas estratégias de atuação acadêmica têm abordagens interdisciplinares e voltadas à Saúde Pública.

\section{Metodologia}

O protocolo de pesquisa foi aprovado pelo Comitê de Ética em Pesquisa da Universidade Estadual de Goiás (parecer consubstanciado número 3.947.882). Foi realizado um estudo do tipo transversal, de abordagem quantitativa e qualitativa. A coleta de dados foi realizada via e-mail utilizando a plataforma Google Forms e participaram da pesquisa os professores na ativa do curso de Bacharel em Fisioterapia de Universidades Estadual de Goiás - UnU ESEFFEGO no ano de 2020. Foi aplicado um questionário misto aos professores, com perguntas abertas, fechadas e de múltipla escolha, com o intuito de compreender como o docente lida com a abordagem da Saúde Pública e o trabalho interdisciplinar. As questões visaram compreender o entendimento dos docentes em relação à sua perspectiva diante do SUS e como é a atuação do fisioterapeuta e sua integração com outras disciplinas e áreas da saúde. Os professores foram previamente orientados sobre a pesquisa durante reunião de colegiado do curso e os questionários foram enviados para que cada um respondesse no período determinado.

Para a análise foi utilizado o método de conteúdo com destaque para a abordagem qualitativa da pesquisa social (Minayo \& Gomes, 1994). Foi realizada a leitura dos instrumentos preenchidos pelos professores buscando uma compreensão global dando ênfase ao conteúdo e a lógica, já que a frequência de aparição pode ter um significado para o objetivo analítico estudado. No primeiro momento da análise foi realizada a leitura flutuante dos documentos, com a reunião e conferência dos dados coletados. A seguir foi feita a leitura longitudinal das respostas do questionário misto, identificando as características comuns ou que se relacionam entre si e por fim o tratamento dos resultados e sua interpretação com uma leitura transversal do material caracterizado.

\section{Resultados e Discussão}

Foram encaminhados 42 questionários e 24 docentes retornaram o convite com as respostas. Os entrevistados foram interrogados inicialmente quanto aos mecanismos utilizados para melhora no exercício da docência. As respostas estão apresentadas na Tabela 1. A prática docente no ensino superior no Brasil, de maneira geral, tende a iniciar-se sem preparação prévia e de modo intuitivo. Logo, esses professores são formados na prática cotidiana passando por constantes transformações e aprendizados (Pereira \& Anjos, 2014). Assim, a reflexão sobre como aperfeiçoar a técnica de ensino é um exercício de importância extrema. Para análise quantitativa o programa Excel foi o utilizado para cálculo de frequências. 
Tabela 1: Mecanismos utilizados para melhora no exercício da docência.

\begin{tabular}{|c|c|}
\hline Perguntas & $\begin{array}{l}\text { Frequência } \\
\text { das Respostas }\end{array}$ \\
\hline $\begin{array}{l}\text { Simplesmente ministra as aulas e faz ajustes sucessivos e adaptação } \\
\text { progressiva de comportamentos }\end{array}$ & $12,5 \%$ \\
\hline Combina ação e reflexão sobre a ação & $29,1 \%$ \\
\hline Faz reflexão retrospectiva sobre a ação & $16,6 \%$ \\
\hline Faz uma reflexão antecipadora de mudança sobre a ação & $16,6 \%$ \\
\hline $\begin{array}{l}\text { Faz a aquisição de saberes teóricos por integração/assimilação de saberes } \\
\text { novos }\end{array}$ & $25 \%$ \\
\hline Não faz essa reflexão & 0 \\
\hline
\end{tabular}

Fonte: Autores (2021).

Em relação aos trabalhos anteriores e atuais dos entrevistados, além de exercerem a função de docente, destacaram-se as funções de fisioterapeuta, pesquisador e palestrante. As áreas de interesse dos participantes são diversas e passam por variados campos da fisioterapia, desde terapias manuais a cardiologia. Essa variedade de campos de atuação é apontada por Badaró; Guilhem em 2011 como consequência da evolução da fisioterapia, de uma profissão com foco de na reabilitação para profissão autônoma e com espaço ampliado para atuação (Badaró \& Guilhem, 2011).

Contudo, as respostas quanto as perspectivas e dificuldades são semelhantes. A perspectiva de crescimento é citada em muitas respostas, já com relação as dificuldades aparecem em comum a dificuldade de se inserir na área almejada e a falta de retorno financeiro.

"[...] limitação de oportunidades e carência de boas opções de trabalho e reconhecimento"

“[...] Não valorização profissional em início de carreira”

“[...] perspectivas desenvolver trabalhos e pesquisas na área e dificuldades o reconhecimento financeiro e de espaço físico para desenvolver o trabalho. Há também falta de recursos e investimentos em pesquisa e projetos de atuação"

Uma pesquisa realizada por Almeida e Guimarães no ano de 2009 (Almeida \& Guimarães, 2009) demonstrou que a insatisfação do profissional fisioterapeuta está associada ao pequeno reconhecimento social, a saturação do mercado e aos baixos salários médios .

A visão dos participantes também é parecida com relação a atual assistência fisioterapêutica. Sendo considerada uma área necessária, crescente e de muito potencial, que pode atuar em todos os níveis de atenção à saúde. Além disso foi destacado o modelo biopsicossocial como um ponto preciso e positivo para a área. Também foi destacado a relevância do conhecimento do fisioterapeuta para a especificidade do tratamento.

"É necessário um olhar biopsicossocial e mais conhecimento por parte dos fisioterapeutas na especificidade do atendimento..."

“[...] percebo uma grande evolução técnica, de abordagens diagnósticas e uma melhoria na óptica diante do paciente, com uma visão globalizada, sistêmica e não focal baseada do local da queixa. Isso gera uma forma integral na conduta e preocupação não só com a melhora dos sintomas, mas buscar tratar as causas do problema [...]”

O olhar biopsicossocial realçado pelos entrevistados, refere-se a uma forma de abordagem em saúde que considera além dos fatores biológicos aspectos psicológicos e sociais. Os contextos nesse modelo são mais abrangentes e tratam o indivíduo como um todo, não se prendendo apenas a doença (Cardoso et al., 2020). 
O modelo biopsicossocial é tratado por (Castro et al., 2015) como tendo forte associação com a formação dos profissionais. Nesse contexto, a reprodução, de qualquer modelo de tratamento do processo saúde-doença, é reflexo das perspectivas dos docentes no ciclo acadêmico. Logo, para que haja mudanças no tratamento da saúde, com foco biopsicossocial, é preciso haver alterações na forma de ensino da graduação em saúde.

Já a perspectiva futura da assistência fisioterapêutica é bem distinta entre os entrevistados. Alguns apontaram que o crescimento da área como um todo tende a levar a valorização da profissão, com profissionais cada vez mais capacitados e preparados (Ruh, 2018) e apontam que o crescimento da profissão está atrelado as necessidades da sociedade, ressaltam que a formação dos profissionais deve considerar esse fato e também atrelar-se a interdisciplinaridade, interprofissionalidade e a continuidade de formação e aprendizado. Outros participantes acreditam que isso irá ocorrer, mas em ramos específicos da fisioterapia.

"Com o envelhecimento da sociedade e maior cuidados individual com a saúde, a fisioterapia será um recurso de assistência cada vez mais requisitado"

"Cada vez com menos opções de bons empregos apesar do aumento do reconhecimento em algumas áreas"

A tecnologia é de relevância para vários participantes, eles afirmam que este fator tende a substituir o profissional em alguns aspectos, além da ampliação de modalidades como o teleatendimento. Segundo os entrevistados isso corrobora para que futuramente a abordagem fisioterapêutica possa ser rasa e inespecífica.

"Há tendência de substituição de pessoas por máquinas fazendo o trabalho mais braçal e substituição de pessoas por inteligência artificial, fazendo o planejamento terapêutico"

"Acredito que o teleatendimento irá ser aceito como rotina e ficaremos cada vez mais ligados à produção em série e tendo que responder a metas de produção"

Os recursos tecnológicos em fisioterapia auxiliam no monitoramento, na avaliação e no tratamento dos pacientes. A vantagem desses recursos é que o tempo de acompanhamento não é restrito, fazendo uma abordagem prolongada do paciente (Holanda et al., 2018). Atualmente, um campo de abrangente discussão é com foco na chamada telessaúde, que utiliza da tecnologia e telecomunicação para promover assistência aos pacientes (Caetano et al., 2020). Esse campo se mostra como um desafio para os profissionais da fisioterapia, pois demanda muitas adaptações e tira desses profissionais o cerne da fisioterapia, o toque.

Em relação a perspectiva dos docentes ao SUS, a grande maioria destaca sua importância como um sistema que abrange de forma universal todos os cidadãos e a disponibilidade de um acesso à saúde sem nenhum custo, logo veem como indispensável para a população brasileira. Porém, grande parte dos docentes ressalta suas deficiências, citando a falta de investimento, más gestões e sua alta demanda para tão pouco investimento.

"É um sistema importante e necessário, para garantir um direito fundamental do ser humano: saúde."

“[...], apresenta um organograma excelente, no entanto esbarra na burocracia, incompetência e desonestidade do estado."

Segundo o Ministério da Saúde do Brasil (Brasil, 2009), para suprir esta demanda é indispensável ter por base uma equipe de saúde formada por profissionais de diversos setores, capazes de atender as diferentes demandas. 
De acordo com a Estratégia da Saúde Família (ESF), a principal iniciativa de organização da Atenção Primária à Saúde (APS) do SUS, o profissional fisioterapeuta possui formação suficiente, adquirindo as competências necessárias durante sua formação para atuar em todos os níveis de atenção. Na visão dos docentes sobre a atuação do fisioterapeuta no SUS, destaca-se a necessidade de a fisioterapia estar mais presente nesse quesito, grande parte descreve que a fisioterapia está presente de forma discreta, atuando basicamente na atenção terciária, destacando a importância da atuação em todos os âmbitos, tanto primária quanto secundária.

“A atuação do fisioterapeuta ainda é muito discreta. Infelizmente nós atuamos basicamente, na fase terciária de atenção à saúde, precisamente na reabilitação no âmbito ambulatorial."

"Atuação importante, mas ainda com poucas oportunidades."

No quesito de habilidades e competências necessárias para o fisioterapeuta trabalhar no SUS, os docentes destacaram a importância do conhecimento sobre este sistema, e relataram ainda a necessidade de profissionais como estes conhecerem melhor o SUS e suas áreas de atuação, descreveram também a importância da formação humanística para trabalhar neste sistema e o conhecimento principalmente sobre atenção primária. Nos estudos de Ribeiro e Flores (2015), verificou-se a falta de conhecimento dos gestores sobre atuação do fisioterapeuta na atenção primária, sendo restringida apenas a atenção terciária. Em contrapartida, os gestores que possuíam fisioterapeutas dentro de suas equipes, identificaram o profissional como promotor de saúde e grande parte destacou a dificuldade de inserir este profissional em outras áreas de atenção, já que o investimento é pequeno e seria difícil o mesmo atender a demanda da atenção terciária (Ribeiro \& Flores-Soares, 2015).

Sobre a integração entre disciplinas e outras áreas da saúde, apenas dois dos participantes relataram que a integração é pouca. Assim, os demais professores descreveram uma grande integração, citaram também a importância do trabalho do fisioterapeuta integrado a outros profissionais da saúde como médicos, enfermeiros, nutricionistas, biomédicos e todas as outras profissões da área da saúde, destacando a importância do trabalho em conjunto destes profissionais, principalmente em âmbito hospitalar e atenção básica.

O fisioterapeuta é formado para atuar nos diversos níveis de assistência à saúde, com as mesmas condições dos demais membros da equipe, com objetivo de preservar, promover, aperfeiçoar ou adaptar, por meio de uma relação terapêutica, o indivíduo a uma melhor qualidade de vida tanto em nível individual quanto coletivo (Oliveira et al., 2011). Cabe ao fisioterapeuta ainda, no trabalho em equipe multiprofissional contribuir para o bem-estar da comunidade, agindo como um multiplicador de saúde. Atualmente, a composição mínima da equipe de Saúde da Família não apresenta o fisioterapeuta como profissional obrigatório para compô-la, porém, de acordo com as necessidades da população, o gestor municipal tem a decisão de poder integrar outros profissionais a esta área (Brasil, 2006).

\section{Conclusão}

Entende-se que a Fisioterapia é uma profissão ampla, com diversas áreas de atuação, necessária e em constante crescimento. A profissão caminha para mudanças, concomitante as necessidades sociais inovações tecnológicas. Apesar disso os profissionais da área sentem-se pouco reconhecidos e mal remunerados. Fato é que a atuação prática da fisioterapia é reflexo direto do aprendizado acadêmico e por isso os docentes da área devem e tentam buscar melhoras no exercício do ensino.

No que se refere ao SUS, o que se pode concluir é que é necessário mais investimentos e integração obrigatória deste profissional em uma equipe multiprofissional de atenção básica, também é necessário um melhor preparo dos profissionais 
fisioterapeutas dentro desta área durante sua formação, e a necessidade do conhecimento sobre a atuação do fisioterapeuta na atenção primária, tanto pelos próprios profissionais quanto pelos gestores governamentais.

Todos estes fatos foram atestados pelos entrevistados desta pesquisa, o que demonstra que o exercício do docente exige competências que envolvam a interdisciplinaridade. Além disso, é fundamental que tenham papel significativo na construção dos saberes e manejos que vão refletir na Saúde Pública. Logo, ao professor fisioterapeuta cabe a elaboração de estratégias que vão preparar o estudante para atuar na sociedade considerando a realidade e suas necessidades. Diante desse cenário e após a realização desse estudo, foram levantadas questões e reflexões a serem abordadas em pesquisas futuras e que possam incrementar a atuação do docente fisioterapeuta, com maior direcionamento às abordagens interdisciplinares e voltadas à Saúde Pública.

\section{Referências}

Almeida, A. L. J., \& Guimarães, R. B. (2009). O lugar social do fisioterapeuta brasileiro. Fisioterapia e Pesquisa, 16(1), 82-88. https://doi.org/10.1590/s180929502009000100015

Badaró, A. F. V., \& Guilhem, D. (2011). Perfil sociodemográfico e profissional de fisioterapeutas e origem das suas concepções sobre ética. Fisioterapia em Movimento, 24(3), 445-454. https://doi.org/10.1590/s0103-51502011000300009

Bispo Júnior, J. P. (2010). Physiotherapy and collective health: Challenges and new professional responsibilities. Ciencia e Saude Coletiva, 15(SUPPL. 1), 1627-1636. https://doi.org/10.1590/s1413-81232010000700074

Brasil. Ministério da Saúde. (2006). Política nacional de promoção da saúde

Brasil. Ministério da Saúde. (2009). Cadernos de Atenção Básica

Caetano, R., Silva, A. B., Guedes, A. C. C. M., Paiva, C. C. N., Rocha Ribeiro, G., Santos, D. L., \& Silva, R. M. (2020). Challenges and opportunities for telehealth during the COVID-19 pandemic: Ideas on spaces and initiatives in the Brazilian context. Cadernos de Saude Publica, 36(5), 1-16. https://doi.org/10.1590/0102-311X00088920

Cardoso, K. V. C. V., Ferreira, B. A., \& Castro, S. S. (2020). Utilização do modelo biopsicossocial no estudo da anatomia humana no curso ee Fisioterapia: relato de experiência. Cadernos De Educação, Saúde E Fisioterapia, 6(11). https://doi.org/10.18310/2358-8306.v6n11.a5

Carvalho, G. (2013). Saúde pública no Brasil. Estudos Avançados, 27(78), 5-26.

Carvalho, V. L., Tomaz, J. M. T., \& Tavares, C. H. F. (2018). Interprofissionalismo e interdisciplinaridade na formação acadêmica: a percepção dos formandos em fisioterapia. Revista de Enfermagem UFPE on line, 12(4), 908. https://doi.org/10.5205/1981-8963-v12i4a230195p908-915-2018

Castro, S. S., Silva, Y. H. G., Leite, C. F., \& Bollela, V. R. (2015). O processo saúde-doença e o modelo biopsicossocial entre supervisores de um curso de Fisioterapia: estudo qualitativo em uma universidade pública. Cadernos De Educação, Saúde E Fisioterapia, 2(3), 23-38. https://doi.org/10.18310/23588306.v2n3p23

Holanda, L. J., Silva, P. M. M., Lima, J. A., \& Morya, E. (2018). Instrumentação De Recursos Tecnológicos No Processo De Formação Do Fisioterapeuta. In Saberes e competências em fisioterapia (Número January 2020, p. 135-142). https://doi.org/10.22533/at.ed.90118021214

Maia, F. E. S., Moura, E. L. R., Madeiros, E. C., Carvalho, R. R. P., Silva, S. A. L., \& Santos, G. R. (2015). Importância do fisioterapeuta na Atenção Básica de Saúde. Rev Fac Ciênc Méd Sorocaba, 17(3), 110-115. https://doi.org/10.22533/at.ed.44019200814

Minayo, M. C. S., \& Gomes, S. F. D. R. (1994). Pesquisa Social - Teoria, método e criatividade. E. Vozes (25a ed).

Oliveira, G., Andrade, E. S., Santos, M. L., \& Matos, G. S. R. (2011). Conhecimento da equipe de saúde da família acerca da atuação do fisioterapeuta na atenção básica. Rev Bras Promoç Saúde, 24(4), 332-339.

OPAS/OMS, O. P. da S. M. da S. (2000). Funciones Esenciales de Salud Pública. Documento, CD 42/15 XLII Reunión del Consejo Directivo de la Organización Panamericana de la Salud. LII Reunión del Comité Regional de la Organización Mundial de la Salud. Washington, D. C., Septiembre de 2000. $18,24$.

Pereira, L. R., \& Anjos, D. D. (2014). O Professor do Ensino Superior: Perfil, desafios e trajetórias de formação. In Seminário Internacional de Educação Superior 2014 (Vol. 1, Número, p. 1-11).

Ribeiro, C. D., \& Flores-Soares, M. C. (2015). Desafios para a inserção do fisioterapeuta na atenção básica: O olhar dos gestores. Revista de Salud Publica, 17(3), 379-393. https://doi.org/10.15446/rsap.v17n3.44076

Ruh, A. C. (2018). Saberes e Competências em Fisioterapia. In Atena Editora.

Souza, L. E. P. F. (2014). Saúde Pública ou Saúde Coletiva? II Congresso Paranaense de Saúde Pública, 7-21.

Souza, R. R. (2002). O sistema público de saúde brasileiro. Seminário Internacional - tendências e desafios dos sistemas de saúde nas Américas, 45. http://www.saude.sc.gov.br/saudetrabalhador/conferencia_estadual/textos_apoio/O Sistema P\%FAblico de Sa\%FAde Brasileiro(CEST).pdf

Tavares, L. R. C., Costa, J. L. R., Oishi, J., \& Driusso, P. (2018). Inserção da fisioterapia na atenção primária à saúde: análise do cadastro nacional de estabelecimentos de saúde em 2010. Fisioterapia e Pesquisa, 25(1), 9-19. https://doi.org/10.1590/1809-2950/15774625012018 